Journal of Theoretical and Computational Acoustics

Vol. 26, No. 4 (2018) 1850013 (9) pages)

(C) The Author(s)

DOI: $10.1142 / \mathrm{S} 2591728518500135$

\title{
Range-Dependent Seismo-Acoustic Propagation in the Marginal Ice Zone
}

\author{
Joseph M. Fialkowski, Michael D. Collins*, David C. Calvo \\ and Altan Turgut \\ Code 7160, Naval Research Laboratory \\ Washington, D.C. 20375, USA \\ *michael.collins@nrl.navy.mil
}

Received 3 July 2017

Accepted 13 September 2017

Published 7 November 2017

\begin{abstract}
Single-scattering operators are used to extend the seismo-acoustic parabolic equation to problems involving transitions between areas with and without ice cover, which are common in the marginal ice zone. Gradual transitions are handled with single-scattering operators for sloping fluid-solid interfaces. Sudden transitions, which may occur when the ice fractures and drifts, are handled with a single-scattering operator that conserves normal displacement and tangential stress across the vertical interfaces between the range-independent regions that are used to approximate a rangedependent environment. The approach is tested by making comparisons with a finite-element model for problems involving range-dependent features in the ice cover and in a sediment that supports shear waves.
\end{abstract}

Keywords: Parabolic equation method; seismo-acoustics; range dependence; Arctic acoustics; marginal ice zone; elastic sediments.

\section{Introduction}

The parabolic equation method may be used to efficiently obtain accurate solutions to many range-dependent problems in ocean acoustics. 1 This approach is extended to handle transitions between areas with and without ice cover, which are common in the marginal ice zone. A parabolic wave equation is based on a factorization of the operator in the wave equation that is exact for range-independent problems (no horizontal variations in the properties of the medium). Range-dependent problems may be handled by approximating the medium in

This is an Open Access article published by World Scientific Publishing Company. It is distributed under the terms of the Creative Commons Attribution 4.0 (CC BY) License which permits use, distribution and reproduction in any medium, provided the original work is properly cited. 


\section{J. M. Fialkowski et al.}

terms of a series of range-independent regions, using the parabolic wave equation to propagate the field through each region, and applying single-scattering approximations at the vertical interfaces between regions. ${ }^{2}$ Different single-scattering approximations are appropriate for different types of range dependence, which may include sloping interfaces and boundaries and continuous changes within layers. Single-scattering approximations for problems involving sloping fluid--solid interfaces ${ }^{3}$ are used to handle gradual transitions between areas with and without ice cover. The treatment of the fluid-solid interface conditions involves a four-point difference formula,, 4 which requires a careful numerical implementation when an ice layer tapers to zero thickness. Sudden transitions between areas with and without ice cover are handled with a single-scattering approximation that conserves normal displacement and tangential stress. Accuracy is tested by making comparisons with solutions generated using a finite-element model.

\section{Single Scattering}

Parabolic equation techniques for generating an initial condition and for propagating the solution through range-independent regions are discussed in Ref. 6. In this section, we discuss single-scattering operators that are applied to the incident fields at the vertical interfaces between regions in order to obtain approximations for the transmitted fields in the subsequent regions. There does not exist a unique single-scattering approximation for certain types of range dependence, but the approaches discussed here have been found to provide accurate and efficient solutions for a wide range of problems. We consider a two-dimensional problem and work in cylindrical coordinates, with the range $r$ being the horizontal distance from a source and $z$ being the depth below a reference point. The coefficients of the elastic wave equation are defined in terms of the density $\rho$ and the Lamé parameters $\lambda$ and $\mu$. Wave propagation problems are often defined in terms of the wave speeds,

$$
\begin{aligned}
& c_{p}=\sqrt{\frac{\lambda+2 \mu}{\rho}}, \\
& c_{s}=\sqrt{\frac{\mu}{\rho}}
\end{aligned}
$$

which are allowed to be complex in order to account for the attenuations $\beta_{p}$ and $\beta_{s}$ (in decibels per wavelength). As discussed in Ref. 6 , there are advantages in using the dependent variables $u_{x}$, which is the horizontal derivative of the horizontal displacement, and $w$, which is the vertical displacement.

Single-scattering approximations are based on depth operators for converting between the dependent variables and the quantities that are conserved across the vertical interfaces between range-independent regions. With models that are based on separation of variables, it is relatively easy to obtain basic quantities, such as the displacements and stresses, from the dependent variables by using analytic relationships in which operators are equivalent to 
multiplication by separation constants. With the parabolic equation method, these quantities may be obtained using the equations,

$$
\begin{aligned}
\left(\begin{array}{c}
\sigma_{x x} \\
w
\end{array}\right) & =R\left(\begin{array}{c}
u_{x} \\
w
\end{array}\right), \\
\left(\begin{array}{c}
u \\
-\sigma_{x z}
\end{array}\right) & =-i S\left(L^{-1} M\right)^{-1 / 2}\left(\begin{array}{c}
u_{x} \\
w
\end{array}\right), \\
R & =\left(\begin{array}{cc}
\lambda+2 \mu & \lambda \frac{\partial}{\partial z} \\
0 & 1
\end{array}\right), \\
S & =\left(\begin{array}{cc}
1 & 0 \\
\lambda \frac{\partial}{\partial z}+\frac{\partial \lambda}{\partial z} & \frac{\partial}{\partial z}(\lambda+2 \mu) \frac{\partial}{\partial z}+\rho \omega^{2}
\end{array}\right)
\end{aligned}
$$

where the $2 \times 2$ depth operators $L$ and $M$ are defined in Ref. 3. As discussed in Ref. 7, many problems involving sloping solid-solid interfaces may be handled accurately using the single-scattering approximation,

$$
\left(\begin{array}{c}
u_{x} \\
w
\end{array}\right)_{t}=\frac{i}{2}\left(L_{B}^{-1} M_{B}\right)^{1 / 2} S_{B}^{-1}\left(\begin{array}{c}
u \\
-\sigma_{x z}
\end{array}\right)_{i}+\frac{1}{2} R_{B}^{-1}\left(\begin{array}{c}
\sigma_{x x} \\
w
\end{array}\right)_{i},
$$

where the subscripts $i$ and $t$ denote the incident and transmitted fields and the subscripts $A$ and $B$ denote the regions on the incident and transmitted sides of the vertical interface. This approach may be used to handle a sloping solid boundary by placing an artificial layer with low wave speeds and density above the boundary and treating it as a solid-solid interface.

For problems involving sloping fluid-solid interfaces, there are two mathematically distinct cases that call for different approaches. For both of the cases, there is fluid on one side of the rise and solid on the other side. When the solid is on the transmitted side of the rise, accurate solutions may often be obtained with the single-scattering approximation, $\frac{3}{,}$

$$
\begin{aligned}
& \left(\begin{array}{l}
u_{x} \\
w
\end{array}\right)_{t}=\frac{i}{2}\left(L_{B}^{-1} M_{B}\right)^{1 / 2} S_{B}^{-1}\left(\begin{array}{c}
u \\
-\sigma_{x z}
\end{array}\right)_{i}+\frac{1}{2} Q_{B}^{-1}\left(\begin{array}{c}
\sigma_{x x} \\
\frac{-\partial \sigma_{x z}}{\partial x}
\end{array}\right)_{i}, \\
& \left(\begin{array}{c}
\sigma_{x x} \\
\frac{-\partial \sigma_{x z}}{\partial x}
\end{array}\right)=Q\left(\begin{array}{c}
u_{x} \\
w
\end{array}\right) \\
& Q=\left(\begin{array}{cc}
\lambda+2 \mu & \lambda \frac{\partial}{\partial z} \\
\lambda \frac{\partial}{\partial z}+\frac{\partial \lambda}{\partial z} & \frac{\partial}{\partial z}(\lambda+2 \mu) \frac{\partial}{\partial z}+\rho \omega^{2}
\end{array}\right) .
\end{aligned}
$$




\section{J. M. Fialkowski et al.}

Since $\partial \sigma_{x z} / \partial x$ vanishes along the runs, it is small on the rises in the limit of small grid spacing. The incident value of this quantity is therefore set to zero on the rises. When the fluid is on the transmitted side of the rise, accurate solutions may often be obtained by introducing an arbitrarily thin film of solid material with a low shear speed on the faces of the rises and solving two single-scattering problems. The first problem may be solved using Eq. (77) since the rise is a solid-solid interface. The second problem may be solved by using the single-scattering approximation,

$$
\left(\begin{array}{c}
u_{x} \\
w
\end{array}\right)_{t}=i\left(L_{B}^{-1} M_{B}\right)^{1 / 2} S_{B}^{-1}\left(\begin{array}{c}
u \\
-\sigma_{x z}
\end{array}\right)_{i},
$$

which conserves normal displacement and tangential stress. This single-scattering approximation also provides accurate solutions in many cases for problems involving discontinuous changes in ice thickess in which the rise spans several grid points.

Within each range-independent region, layers are separated by the runs of stair steps, which are horizontal interfaces. Horizontal solid-solid interfaces may be handled using Galerkin's method to discretize the depth operators. ${ }^{[6}$ Horizontal fluid-solid interfaces may be handled using an implementation of interface conditions that involves a four-point difference formula! ${ }^{4}$ When using this formula, the solid layer must contain at least three grid points; the fourth grid point is an artificial grid point on the other side of the horizontal interface. In order to avoid the possibility of introducing instabilities, the ice layer is allowed to have three or more grid points (in areas with ice cover) or zero grid points (in areas without ice cover), but it is not allowed to have only one or two grid points. The approach that involves a film of intermediate material on the rises was implemented by requiring that each rise spans four grid points. These numerical approaches produced good results for the test cases, but it may be possible to improve efficiency by introducing nonuniform grid spacing. 4

\section{Test Cases}

Each of the examples involves a $25 \mathrm{~Hz}$ point source in a water column in which the sound speed is $1500 \mathrm{~m} / \mathrm{s}$. All layers are homogeneous and lossless unless specified otherwise. The parameters of the ice are $c_{p}=3500 \mathrm{~m} / \mathrm{s}, c_{s}=1750 \mathrm{~m} / \mathrm{s}, \rho=0.9 \mathrm{~g} / \mathrm{cm}^{3}, \beta_{p}=0.1$, and $\beta_{s}=0.2$. The parameters of the sediment half space are $c_{p}=3400 \mathrm{~m} / \mathrm{s}, c_{s}=1700 \mathrm{~m} / \mathrm{s}, \rho=$ $2.5 \mathrm{~g} / \mathrm{cm}^{3}, \beta_{p}=0.2$, and $\beta_{s}=0.4$. In the films of solid material on the faces of the rises, $c_{p}=1500 \mathrm{~m} / \mathrm{s}, c_{s}=500 \mathrm{~m} / \mathrm{s}$, and $\rho=1 \mathrm{~g} / \mathrm{cm}^{3}$. In the artificial layer of solid material above the water and ice, $c_{p}=200 \mathrm{~m} / \mathrm{s}, c_{s}=100 \mathrm{~m} / \mathrm{s}, \rho=0.001 \mathrm{~g} / \mathrm{cm}^{3}, \beta_{p}=10$, and $\beta_{s}=10$. Reference solutions are generated using a finite-element model ${ }^{[5}$ The transmission loss is defined in terms of $\sigma_{z z}$, which is continuous across horizontal interfaces.

The purpose of examples A and B is to test the parabolic equation solution for problems involving gradual transitions between areas with and without ice cover. For both of the cases, a sloping ice-water interface is the only form of range dependence. For both of the cases, the source is $280 \mathrm{~m}$ below the ice in a water column that is $300 \mathrm{~m}$ deep at $r=0$; the 

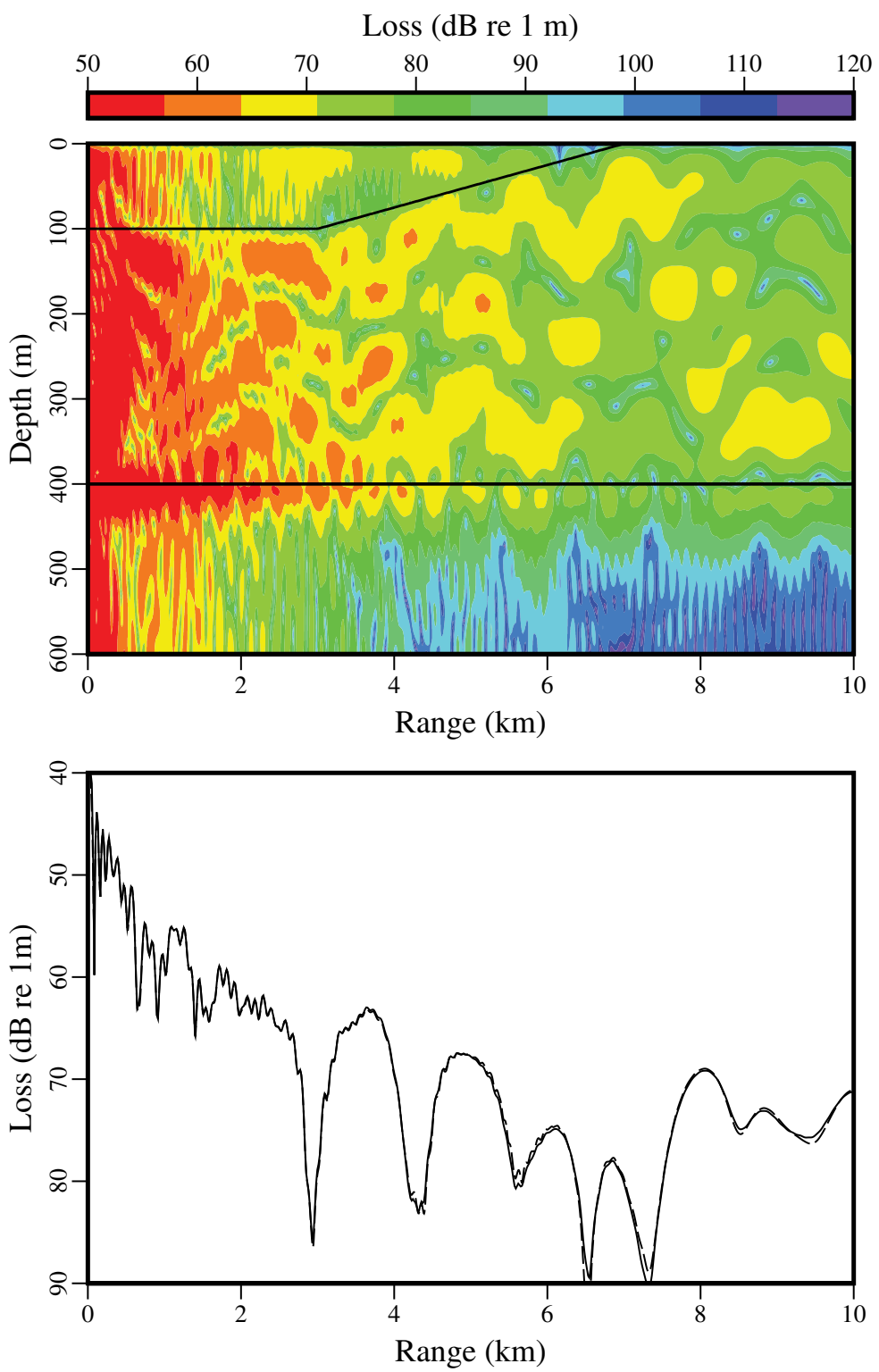

Fig. 1. Transmission loss for example A, which involves a water column with ice cover above for $r<7 \mathrm{~km}$ and an elastic sediment below. The curves in the lower half of the figure correspond to the transmission loss at $z=280 \mathrm{~m}$. The parabolic equation solution (solid curve) is in agreement with the reference solution (dashed curve).

thickness of the ice is $100 \mathrm{~m}$ for $r<3 \mathrm{~km}$; the thickness of the ice linearly decreases to zero for $3 \mathrm{~km}<r<7 \mathrm{~km}$; and there is no ice for $r>7 \mathrm{~km}$. For the downslope case, the source is $280 \mathrm{~m}$ below the surface of a water column that is $300 \mathrm{~m}$ deep at $r=0$; there is no ice for $r<3 \mathrm{~km}$; the thickness of the ice linearly increases to $100 \mathrm{~m}$ for $3 \mathrm{~km}<r<7 \mathrm{~km}$; and the 

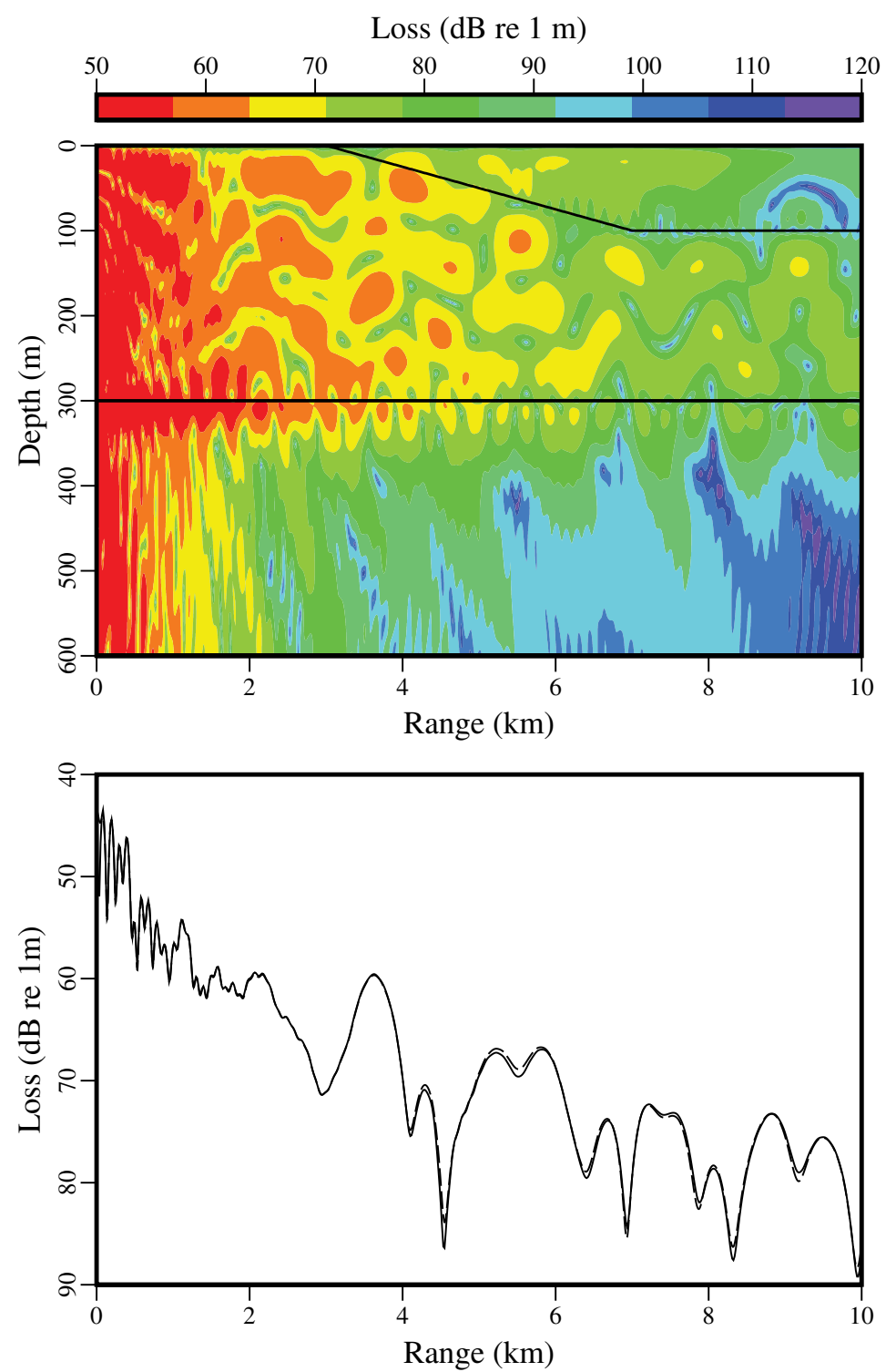

Fig. 2. Transmission loss for example B, which involves a water column with ice cover above for $r>3 \mathrm{~km}$ and an elastic sediment below. The curves in the lower half of the figure correspond to the transmission loss at $z=180 \mathrm{~m}$. The parabolic equation solution (solid curve) is in agreement with the reference solution (dashed curve).

thickness of the ice is $100 \mathrm{~m}$ for $r>7 \mathrm{~km}$. The parabolic equation solutions that appear in Figs. 1 and 2 are in agreement with the reference solutions for both cases.

The purpose of example $\mathrm{C}$ is to test the parabolic equation solution for a problem involving sudden transitions between areas with and without ice cover. The source is $280 \mathrm{~m}$ below the ice in a water column that is $300 \mathrm{~m}$ deep at $r=0$. There is no ice for $3 \mathrm{~km}<$ $r<7 \mathrm{~km}$; outside of this interval, the thickness of the ice is $20 \mathrm{~m}$. The parabolic equation 

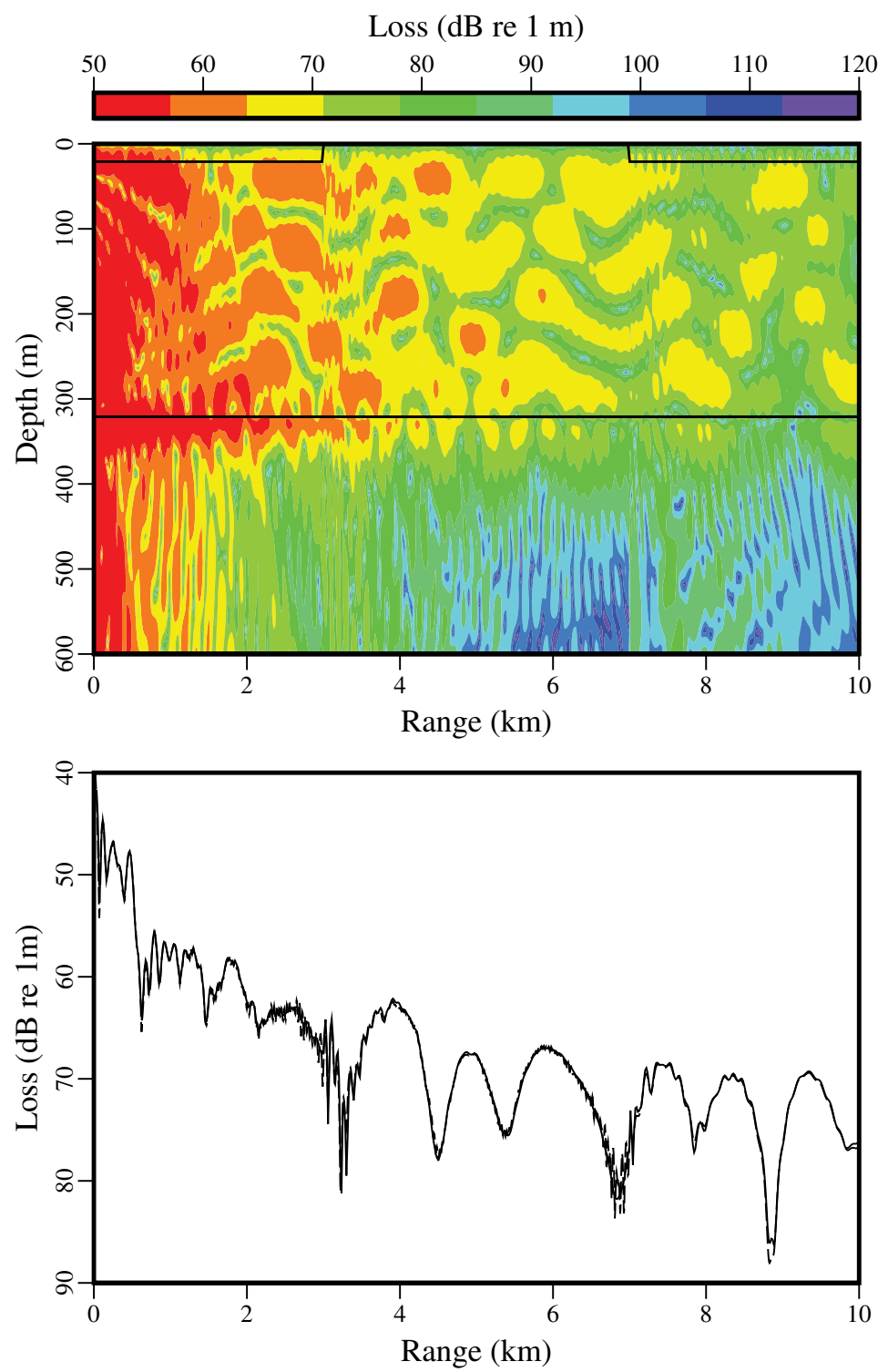

Fig. 3. Transmission loss for example C, which involves breaks in the ice cover at $r=3 \mathrm{~km}$ and $r=7 \mathrm{~km}$ and a water column over an elastic bottom. The curves in the lower half of the figure correspond to the transmission loss at $z=200 \mathrm{~m}$. The parabolic equation solution (solid curve) is in agreement with the reference solution (dashed curve).

solution that appears in Fig. 3 is in agreement with the reference solution. A significant amount of energy that scatters from the edges of the ice appears in the interference pattern in the color plot. This interference pattern also appears in the transmission loss curve near $r=8 \mathrm{~km}$, which indicates that the parabolic equation accurately handles scattering from the edges. 

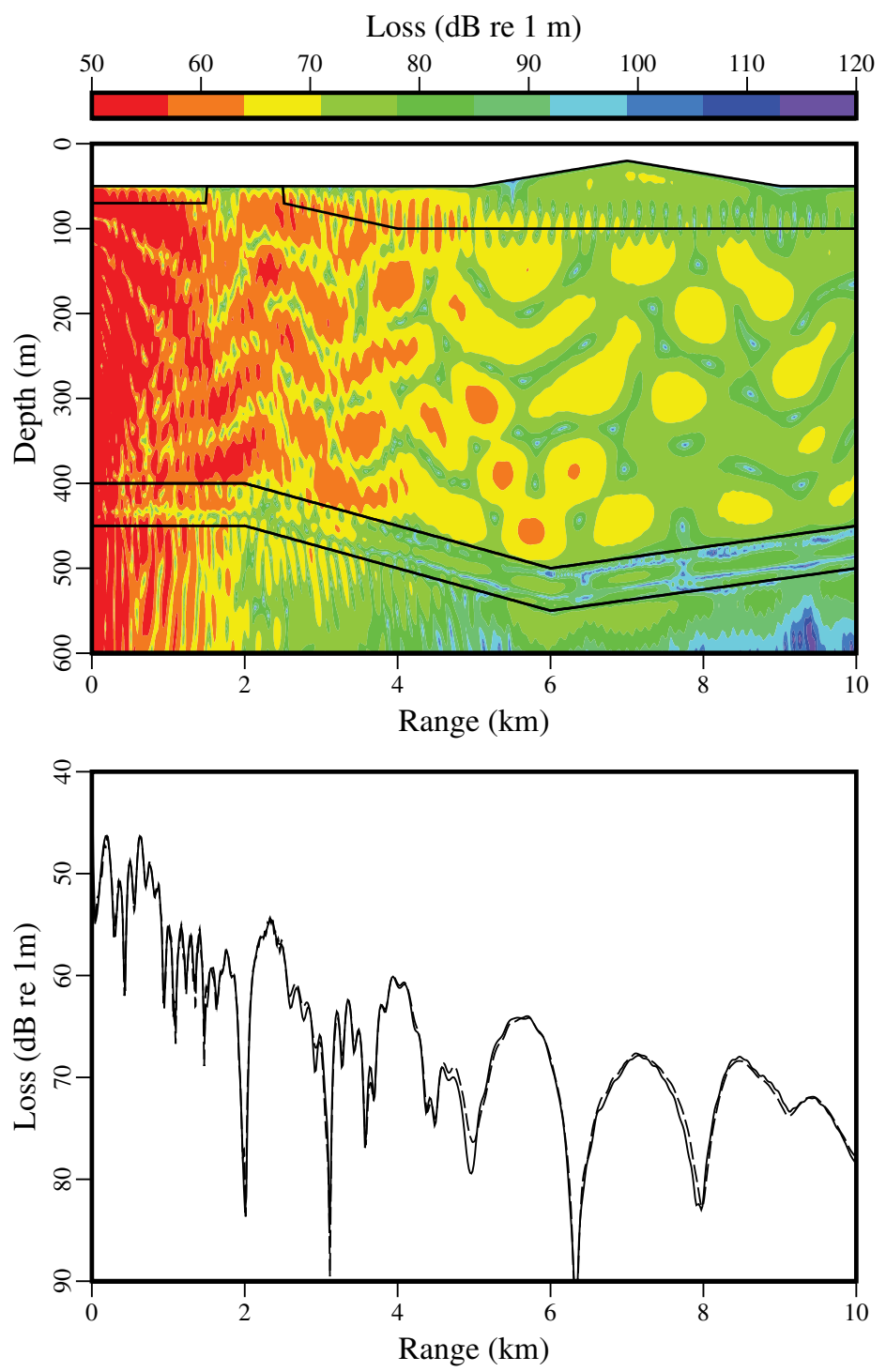

Fig. 4. Transmission loss for example D, which involves several types of range dependence. The curves in the lower half of the figure correspond to the transmission loss at $z=150 \mathrm{~m}$. The parabolic equation solution (solid curve) is in agreement with the reference solution (dashed curve).

The purpose of example $\mathrm{D}$ is to test the parabolic equation solution for a problem involving several types of range dependence, including continuous and sudden transitions between areas with and without ice cover, ice with variable topography, a sloping fluid-solid interface between the water column and an elastic sediment layer, and a sloping solid-solid interface between the sediment layer and the half space. The parameters of the $50 \mathrm{~m}$ thick sediment are $c_{p}=1700 \mathrm{~m} / \mathrm{s}, c_{s}=800 \mathrm{~m} / \mathrm{s}, \rho=1.2 \mathrm{~g} / \mathrm{cm}^{3}, \beta_{p}=0.2$, and $\beta_{s}=0.4$. The source is $310 \mathrm{~m}$ below the ice in a water column that is $330 \mathrm{~m}$ deep at $r=0$. As indicated 
in Fig. 4, the thickness of the ice ranges between 20 and $80 \mathrm{~m}$, and there is no ice cover for $1.5 \mathrm{~km}<r<2.5 \mathrm{~km}$. The parabolic equation solution that appears in Fig. 4 is in agreement with the reference solution.

\section{Discussion}

The seismo-acoustic parabolic equation has been extended to handle problems involving continuous and sudden transitions between areas with and without ice cover. The continuous case is based on single-scattering approximations for sloping fluid-solid interfaces. The sudden case is based on conservation of normal displacement and tangential stress across vertical interfaces. For both cases, Galerkin's method is used to account for piecewise continuous depth dependence within solids, and interface conditions involving a four-point difference formula are used to account for horizontal fluid-solid interfaces. Parabolic equation solutions are in agreement with reference solutions for a variety of problems in Arctic acoustics.

\section{Acknowledgment}

This work was supported by the Office of Naval Research.

\section{References}

1. F. B. Jensen, W. A. Kuperman, M. B. Porter and H. Schmidt, Computational Ocean Acoustics (American Institute of Physics, New York, 1994), p. 612.

2. M. D. Collins, A single-scattering correction for the seismo-acoustic parabolic equation, J. Acoust. Soc. Am. 131 (2012) 2638-2642.

3. K. Woolfe, M. D. Collins, D. C. Calvo and W. L. Siegmann, Seismo-acoustic benchmark problems involving sloping fluid-solid interfaces, J. Comp. Acoust. 24 (2016) 1650022.

4. M. D. Collins and W. L. Siegmann, Treatment of a sloping fluid-solid interface and sediment layering with the seismo-acoustic parabolic equation, J. Acoust. Soc. Am. 137 (2015) 492-497.

5. COMSOL Multiphysics@ v. 5.2 (COMSOL AB, Stockholm, Sweden).

6. W. Jerzak, W. L. Siegmann and M. D. Collins, Modeling Rayleigh and Stonely waves and other interface and boundary effects with the parabolic equation, J. Acoust. Soc. Am. 117 (2005) 3497-3503.

7. K. Woolfe, M. D. Collins, D. C. Calvo and W. L. Siegmann, Seismo-acoustic benchmark problems involving sloping solid-solid interfaces and variable topography, J. Comp. Acoust. 24 (2016) 1650019. 